\title{
Effects of tyrosine kinase inhibition on bone metabolism: untargeted consequences of targeted therapies
}

\author{
José O Alemán*, Azeez Farooki* and Monica Girotra \\ Endocrine Service, Department of Medicine, Memorial Sloan Kettering Cancer Center, 1275 York Avenue, \\ Weill Cornell Medical College, New York, New York 10065, USA \\ *(J O Aleman and A Farooki contributed equally to this work)
}

Correspondence should be addressed to A Farooki

Email

farookia@mskcc.org

\begin{abstract}
Tyrosine kinase inhibitors (TKIs) are at the forefront of molecular-targeted therapies for cancer. With the advent of imatinib for the treatment of chronic myelogenous leukemia, a new wave of small-molecule therapeutics redefined the oncologic treatment to become chronically administered medications with tolerable side-effect profiles compared with cytotoxic agents. Effects on bone mineral metabolism were observed during early imatinib treatment, in the form of hypophosphatemia with increased urinary phosphorus excretion. This finding led to detailed investigations of off-target effects responsible for changes in bone cell maturation, activity, and impact on bone mass. Subsequently, another BCR-Abl inhibitor (dasatinib), vascular endothelial growth factor (VEGF) inhibitors (sorafenib and sunitinib) as well as rearranged during transfection (RET) inhibitors (vandetanib and cabozantinib) were developed. Inhibition of bone resorption appears to be a class effect and is likely contributed by TKI effects on the hematopoietic and mesenchymal stem cells. As long-term, prospective, clinical outcomes data accumulate on these targeted therapies, the full extent of off-target side effects on bone health will need to be considered along with the significant benefits of tyrosine kinase inhibition in oncologic treatment.
\end{abstract}
Key Words
- bone
- calcium
- metastasis
- parathyroid hormone
- tyrosine kinase inhibitor

\section{Introduction}

With the discovery of tyrosine kinase signaling through the insulin receptor in the 1970s, a new field of scientific inquiry and therapeutic opportunity opened that is actively developing into treatments for multiple types of cancer. The discovery of BCR-Abl kinase activation as the pathognomonic translocation in the Philadelphia chromosome in turn marked the era of molecular diagnostics and therapeutics in oncology. Imatinib binds to the ATP-binding site of a select group of protein tyrosine kinases including BCR$\mathrm{Abl}$, thereby preventing ATP-binding and subsequently inhibiting kinase activity. Imatinib is recognized as a paradigm shift from cytotoxic therapy to 'targeted therapy' of molecular alterations in chronic myelogenous leukemia (CML; Buchdunger et al. 1996, Druker et al. 1996). The use of tyrosine kinase inhibitors (TKIs) soon led to observations regarding off-target effects of these medications via nonselective inhibition of additional tyrosine kinases or other pharmacological effects. Changes in bone metabolism, a now well-described side effect of imatinib, were first reported in patients receiving long-term therapy who were noted to have laboratory evidence of hypophosphatemia with concomitant phosphaturia and decreased biochemical markers of both bone formation (osteocalcin) and/or resorption (urine N-telopeptide (NTX)) (Berman et al. 2006). This study

Published by Bioscientifica Ltd 
led to subsequent in vitro and in vivo analyses of the impact of imatinib on bone mineral metabolism. With the development of subsequent BCR-Abl inhibitors, such as dasatinib, hypophosphatemia persisted as a side effect, suggesting a drug class effect on phosphate metabolism due to off-target effects on osteoblasts and osteoclasts. With prospective study of imatinib, decreases in calcium and phosphate, secondary hyperparathyroidism, a transient increase in bone formation markers, and a decrease in bone resorption markers were noted (O'Sullivan et al. 2009). Newer generation TKIs (VEGFi and RETi) or 'multikinase' inhibitors for disease processes as varied as CML to thyroid cancer may also have off-target effects on bone metabolism that will need to be elucidated through molecular and cellular studies with attention also to long-term clinical outcomes (Sherman 2009).

The following review briefly summarizes the known pathophysiology of the tyrosine kinases in bone metabolism as they relate to TKI therapy, focusing on BCR-Abl kinase inhibitors as the founding example and expanding to the additional targets vascular endothelial growth factor (VEGF) and rearranged during transfection (RET). Subsequently, we will cover preclinical and clinical data on known inhibitors of these targets, highlighting clinical outcomes relating to bone metabolism data whenever possible or available. Lastly, we shall develop general principles from the studied TKIs to be applied to development of future agents. We postulate that the growth inhibition targeted by many TKIs may inhibit bone formation as well as bone resorption. Long-term safety data with each agent class should ideally include studies of bone mineral metabolism and bone mineral density (BMD, a strong predictor of fracture risk) with attention to adverse events indicative of bone fragility to assess the impact of each agent and class.

\section{Tyrosine kinases in bone metabolism}

\section{BCR-Abl}

BCR-Abl is the tyrosine kinase found in the pathognomonic Philadelphia chromosome in CML. The BCR-Abl tyrosine kinase results from the translocation of chromosomes 9 and 22 leading to the BCR promoter constitutively activating Abl kinase subsequently producing a continued proliferative signal (Druker et al. 1996). Although imatinib works to inhibit this kinase, BCR-Abl is not thought to be involved in bone metabolism per se. Berman et al. (2006) first reported the occurrence of imatinib-treated patients with hypophosphatemia compared with healthy controls. These findings were accompanied by increases in serum parathyroid hormone (PTH) levels; 25 hydroxyvitamin D (25-OH D) levels were normal in six out of the total sixteen hypophosphatemic patients. Imatinib is thought to influence bone mineral metabolism via inhibition of other tyrosine kinases present on bone cells, including platelet-derived growth factor $\alpha$ (PDGFR $\alpha$ ) and PDGFR $\beta$, C-kit, and the C-FMS receptor on monocytes and macrophages as described below.

C-kit C-kit is a transmembrane tyrosine kinase and its activation is implicated in the pathogenesis of gastrointestinal stromal tumors (GIST). C-kit binds to ligand stem cell factor (SCF) to transduce along the mitogenic pathways of microphthalmia transcription factor (MitF) (Huang et al. 1992). $\mathrm{MitF}^{-1-}$ mice show a phenotype of severe osteopetrosis from defective osteoclasts. SCF has been found to stimulate osteoclast precursor proliferation in vitro leading to increase osteoclast numbers (Steingrímsson et al. 1994). The inhibition of c-kit may decrease the number of preosteoclasts as well as inhibit the activity of osteoclasts.

Platelet-derived growth factor PDGF stimulates platelet and cellular proliferation in mesenchymal-derived cells. The corresponding receptor signals through at least two subtypes ( $\alpha$ and $\beta$ ) as a tyrosine kinase with downstream effects on the MAPK pathway. The activation of PDGFR is also implicated in the pathogenesis of GIST as is c-kit. In bone metabolism, PDGF stimulates osteoclastogenesis and subsequent bone resorption in mouse calvaria and iliac crest-derived primary human osteoclasts (Hock 1994, Zhang et al. 1998). These effects are thought to be mediated by stimulating the production of osteoclastogenic cytokines, including receptor activator of nuclear factor $\kappa \mathrm{B}$ ligand (RANKL) and macrophage colonystimulating factor (M-CSF). PDGFR induces osteoblast activity and proliferation in vitro, but these observations have not translated to in vivo evidence of bone formation by serum markers or bone density (Goodkin \& Pierce 1993).

SRC SRC kinase is a soluble tyrosine kinase that is present in normal cells and constitutes the first proto-oncogene described. Its mutated form is present in avian sarcomas and drives proliferation, motility, and adhesion of normal and cancer cells. With respect to bone metabolism, SRC kinase stimulates osteoclastogenesis, osteoclastic activity, and survival, leading to increased bone resorption. The $\mathrm{SRC}^{-1-}$ mouse manifests osteopetrosis, indicating decreased osteoclastic activity. Ruffled borders, a cellular characteristic of mature osteoclasts, are present, but there is failure to resorb bone (Soriano et al. 1991).

Published by Bioscientifica Ltd. 
Macrophage colony-stimulating factor M-CSF is a secreted cytokine that causes hematopoietic stem cells to differentiate in to macrophages through action on its receptor tyrosine kinase (M-CSFR) also known as c-fms. M-CSF is released by osteoblasts upon PTH stimulation and is a key mediator of osteoclast proliferation and differentiation (Horowitz et al. 1989). Imatinib has been shown to potently inhibit the activation of the colonystimulating factor-1 (c-fms) receptor by M-CSF in human bone marrow mononuclear cells as shown in Fig. 1 (Dewar et al. 2005).

\section{Vascular endothelial growth factor}

As an important component of the angiogenic pathway, VEGF is targeted in cancer in order to decrease perfusion of tumor vasculature. In skeletal development, VEGF mediates blood vessel formation and the vascularization of cartilage into bone (Zelzer et al. 2002, Cher et al. 2006, Schipani et al. 2009). VEGF expression is particularly important in the development of various normal tissues such as bone cartilage as well as in tumor development through the hypoxia inducible factor pathway (Maes et al. 2012). VEFGR is present on both osteoblasts and osteoclasts.

\section{Rearranged during transfection}

RET kinase is a tyrosine kinase receptor for a family of neurotrophic ligands. As a protooncogene, its mutation is present in nearly all familial forms of medullary thyroid cancers (MTCs). RET is present in the hematopoietic stem

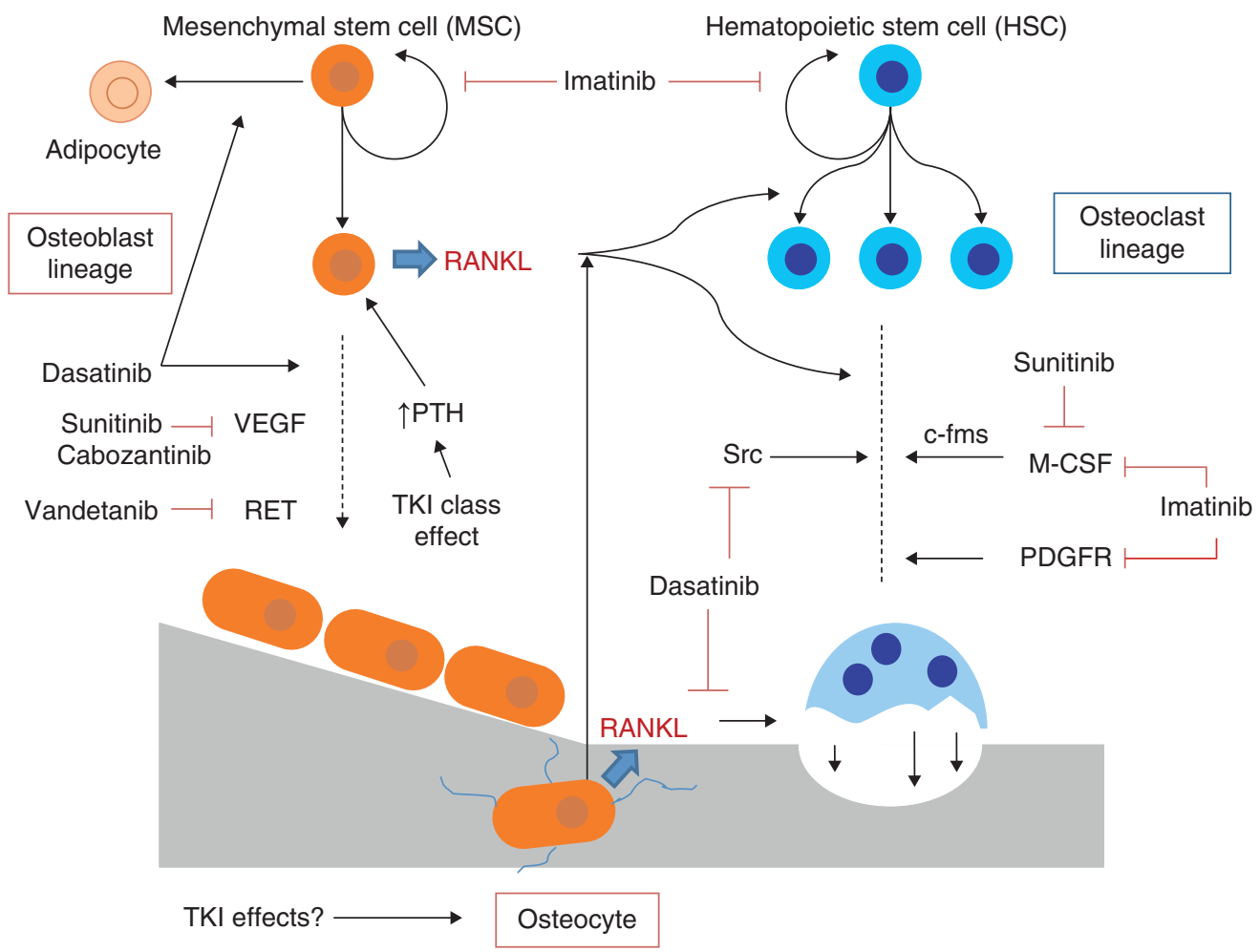

\section{Figure 1}

Effects of tyrosine kinase inhibitors (TKIs) on osteoblast and osteoclast lineages. Osteoblasts are derived from mesenchymal stem cells (MSCs) imatinib inhibits proliferation of MSCs. Dasatinib appears to direct differentiation away from the adipocyte lineage and toward the osteoblast lineage. Inhibitors of VEGF (sunitinib and cabozantinib) and of RET (vandetanib) have been shown to impinge on the osteoblast differentiation process in vitro. Terminally differentiated osteoblasts are known as osteocytes and, along with immature osteoblasts, secrete RANKL, the key driver of osteoclast differentiation and activity. The effects of TKIs on osteocytes are unknown. Tonic elevations in PTH cause an increase in RANKL production, which is expected to cause an increase in osteoclast differentiation and activity. However, despite hyperparathyroidism, several TKIs have been shown to inhibit osteoclast activity. Osteoclasts are derived from hematopoietic stem cells in the bone marrow and stimulated via factors including RANKL to differentiate into mature multinucleated cells. Src kinase has been shown to promote osteoclast differentiation and activity; dasatinib is a potent inhibitor of Src kinase. M-CSF induced differentiation of pre-osteoclasts into mature osteoclasts has been shown to be inhibited by sunitinib and imatinib. Adapted, with permission, from Vandyke K, Fitter S, Dewar AL, Hughes TP \& Zannettino ACW 2010a Dysregulation of bone remodeling by imatinib mesylate. Blood 115 766-774. http://erc.endocrinology-journals.org DOI: $10.1530 /$ ERC-12-0400
(C) 2014 Society for Endocrinology Printed in Great Britain 
cell niche and has been reportedly present in the osteoblast cell lines supporting this microenvironment (Gattei et al. 1997). As a driver of the multiple endocrine neoplasia type 2A (MEN2A) syndrome and MTC, RET is associated with hyperparathyroidism occurring in 10-30\% of cases after the third decade of life. It is thought that stimulus to parathyroid cell proliferation in MEN2A is related to the expression of the mutant RET protein in parathyroid tissue (Pausova et al. 1996).

\section{Tyrosine kinase inhibitors}

\section{Imatinib}

Imatinib was initially discovered as part of a smallmolecule screen for tyrosine kinase activity and subsequently found with specificity for BCR-Abl kinase. Given the Philadelphia chromosome's unique role in the pathophysiology of CML, imatinib emerged as a highly specific molecular treatment for the disorder. However, at treatment doses, this medication has also been shown to inhibit a variety of tyrosine kinases including c-kit, M-CSF (via c-fms receptor), and PDGFR $\alpha$ and PDGFR $\beta$. Before the advent of imatinib, CML was uncommonly associated with a variety of skeletal manifestations including diffuse osteoporosis, osteolytic, and osteoblastic lesions as well as chloromas (Schabel et al. 1980). In chronic phase CML, these skeletal manifestations are generally not present. The effects of imatinib on bone metabolism have been extensively studied and summarized in an excellent review by the Zannettino group (Vandyke et al. 2010a). The following sections describe the effects of imatinib on bone mineral metabolism.

Altered phosphorus metabolism Berman et al. (2006) first described altered phosphorus metabolism in patients receiving imatinib. Clinical trial data suggested a potential side effect of hypophosphatemia, which in turn was confirmed in 16 of 24 patients receiving pharmacological doses of imatinib for the treatment of CML or GIST. Correlation analysis showed that hypophosphatemia was associated with low serum levels of 25-OH D $(P=0.005)$, 1,25-dihydroxyvitamin D (1,25-OH D) $(P=0.04)$, and calcium $(P=0.009)$; however, the number of observations was small.

On the basis of nonspecific tyrosine kinase effects, the authors proposed a model whereby imatinib effects on PDGFR $\alpha$ and PDGFR $\beta$ led to decreased osteoblast and osteoclast activity respectively. Subsequent studies on imatinib showed prospective changes in phosphate metabolism in up to $50 \%$ of patients treated for GIST and CML.

Renal tubular reabsorption of phosphate plays a key role in overall phosphate balance, and is likely the major contributing factor to the hypophosphatemia observed in imatinib-treated patients vs other causes of hypophosphatemia such as decreased gut absorption or acute shift of extracellular phosphate into the cells (i.e. hungry bone syndrome or refeeding). Although it has also been hypothesized that increased bone formation due to imatinib could contribute to hypophosphatemia (Vandyke et al. 2010a), evidence for a sustained increase in bone formation/mineralization is lacking. One prospective study of CML patients reported that maximal tubular resorption of phosphate was consistently decreased relative to baseline levels over 18 months along with mild elevations in PTH levels relative to baseline that stayed in the normal range (O'Sullivan et al. 2009). This overall clinical scenario was consistent with secondary hyperparathyroidism with hypophosphatemia from decreased renal tubular phosphate reabsorption. This inappropriately high urinary phosphate in the face of hypophosphatemia could theoretically be mediated by any of the following: elevated PTH levels, renal tubular damage/dysfunction, fibroblast growth factor 23 (FGF23), or conceivably through other unknown off-target effects of kinase inhibition. The levels of the phosphaturic protein FGF23 in subsequent studies were not elevated in imatinib-treated patients (O'Sullivan et al. 2009) and the expected biochemical profile of FGF23-mediated hypophosphatemia (low levels of 1,25-dihydroxyvitamin $\mathrm{D}_{3}$ and normal PTH levels) has not been demonstrated. The authors found no evidence of Fanconi's syndrome (urinary glucose and amino acid levels were undetectable in all samples at baseline and 3 months). One case report of imatinibinduced partial Fanconi's syndrome with mild renal failure exists (François et al. 2008); another recent report of 20 imatinib-treated (for an unclear duration) CML patients found hypophosphatemia, a relative increase in PTH levels from baseline, and phosphaturia along with a novel finding along with the novel finding of nonselective aminoaciduria without proteinuria, glycosuria, or overt renal failure (urinary $\mathrm{pH}$ not reported) - possible 'partial Fanconi's syndrome' (Ianotto et al. 2012). In summary, secondary hyperparathyroidism contributes to the hypophosphatemia and renal phosphate wasting seen with imatinib, although the relatively small increase in PTH makes it is improbable that secondary hyperparathyroidism is the entire explanation for hypophosphatemia; whether partial Fanconi's syndrome contributes is an open question.

Published by Bioscientifica Ltd. 
Clinically, in adults, long-term negative phosphate balance could result in osteomalacia. Osteomalacia has not been reported in the literature. The diagnosis of Fanconi's syndrome should be ruled out in any patient with low phosphate and phosphaturia. Nutritional vitamin D deficiency should be corrected in all imatinibtreated patients as it may ameliorate hypophosphatemia and hyperparathyroidism. Severe hypophosphatemia should be treated with calcitriol rather than with oral phosphate supplements, which have the potential to bind dietary calcium.

Altered calcium metabolism Relative hypocalcemia as compared with baseline levels with secondary hyperparathyroidism appears to be a pattern with imatinibtreated patients. Berman et al. (2006) found that calcium levels were i) significantly lower in all imatinib treated patients compared with controls and ii) among all imatinib-treated patients, significantly lower in the hypophosphatemic group compared with patients with normal phosphorus levels. As expected physiologically, PTH levels were higher in the imatinib-treated patients with lower calcium levels than those with normal calcium levels. In subsequent prospective investigation, imatinibtreated patients have also been confirmed to manifest a decrease in calcium levels with an associated increase in PTH levels (secondary hyperparathyroidism; O'Sullivan et al. 2009). Another prospective investigation by our group has also shown secondary hyperparathyroidism by and large unrelated to vitamin D deficiency or chronic renal insufficiency (Berman et al. 2013).

Serum calcium is tightly regulated via PTH and vitamin $\mathrm{D}$ via effects on bone, kidney, and the gastrointestinal tract. The expected effect of a decrease in ionized calcium is an immediate increase in PTH levels. PTH in turn works to restore the ionized calcium via the following mechanisms: increased renal calcium reabsorption in the distal tubule, increased intestinal calcium absorption via increased renal production of 1,25-OH D (calcitriol), and an increase in bone resorption/calcium efflux from bone. The causes of hypocalcemia with elevations in serum PTH levels include i) PTH resistance or target organ (kidney and bone) unresponsiveness to PTH as well as, ii) vitamin D deficiency or resistance, iii) chronic kidney disease, or iv) extravascular deposition (via deposition in tissues or by binding within the vasculature). PTH resistance (impaired PTH action) is characterized by hyperphosphatemia and thus is unlikely to contribute. Vitamin D deficiency may contribute to secondary hyperparathyroidism in some cases, although many imatinib-treated patients display persistent secondary hyperparathyroidism in the face 25-OH D levels adequate to suppress PTH levels. Vitamin D resistance via increased metabolism to inactive metabolites is an uninvestigated possibility. This has been described with phenytoin, a hepatic enzyme inducer, although imatinib does not have this property. Vitamin D resistance via decreased 1-hydroxylation of calcidiol to calcitriol $(1,25-\mathrm{OH} \mathrm{D})$ in the kidney appears unlikely given that $1,25-\mathrm{OH} \mathrm{D}$ levels are not low in imatinib-treated patients (O'Sullivan et al. 2009). Vitamin D resistance via decreased calcitriol action is another possibility not yet investigated. Potentially, this could involve a direct effect of kinase inhibition to decrease calcium absorption via impaired calcitriol action. In some patients, poor gut absorption of calcium could also be due to adverse gastrointestinal effects (nausea and diarrhea) which are significant side effects of imatinib.

Long-term studies have provided some data on the effects of imatinib-induced decreases in serum calcium levels, secondary hyperparathyroidism, and phosphaturia on BMD and fracture risk. Recent 2-year prospective BMD data has demonstrated that $\sim 50 \%$ of these patients develop decreases in BMD at the femoral neck and/or total hip, while maintaining stability at the lumbar spine; the distal radius was not evaluated (median duration of imatinib 31 months; range 1-71 months; Berman et al. 2013). Similarly, another group has shown a site-specific decrease in BMD at the femoral neck 24 months after initiation of imatinib, albeit with an associated increase in trabecular bone volume (Vandyke et al. 2012). Thus, given the stability seen at the spine, a site of trabecular bone, as compared with the femoral neck (both cortical and trabecular bone), imatinib might disproportionately affect cortical bone. Arguing against a preferential decrease at cortical sites is the data from the Vandyke group indicating a small increase in BMD at the distal radius over 24 months of follow-up.

Given the small numbers of patients treated in the published studies to date, it is impossible to discern whether imatinib has any effect on fracture risk. One premenopausal patient treated at Memorial Sloan Kettering Cancer Center (MSKCC) with imatinib sustained an unexplained stress fracture of the hip which subsequently healed; biochemistry (including evaluation for secondary causes of bone fragility) and bone density were stable and unrevealing (unpublished data). This patient had no clinical evidence of osteomalacia. There is one reported case of bilateral subtrochanteric fractures in a 60-year-old female with CML who had received imatinib for 1 year. These insufficiency fractures had a similar anatomic

Published by Bioscientifica Ltd 
location, appearance, and prodrome (thigh pain) to 'atypical' fractures reported after long-term bisphosphonate therapy. The patient had normal BMD and evidence of decreased bone mineral apposition rate and loss of double-line tetracycline on bone biopsy (evidence of severely suppressed bone turnover; Yang et al. 2010). No definite conclusions can be drawn from these isolated cases.

We suggest that patients receiving imatinib ingest an amount of calcium and vitamin D from diet and/or supplements in accordance with current guidelines which may help to reduce secondary hyperparathyroidism (IOM 2011). In addition, if hyperparathyroidism is detected, the full differential diagnosis of causes should be explored.

Effects on bone cells Osteoclasts Osteoclasts differentiate from hematopoietic stem cells into peripheral monocyte precursors from the macrophage lineage, forming multinuclear cells responsible for removing bone matrix through resorption (Fig. 1). There is now clear in vitro and in vivo evidence that imatinib decreases the ability of osteoclasts to resorb bone. The in vitro evidence for an anti-osteoclastic activity of imatinib ranges from murine systems showing a decrease in osteoclastic precursors of the hematopoietic stem cell lineage to decreased osteoclastogenesis in human CD14+ peripheral mononuclear cells (Ando et al. 2006, El Hajj Dib et al. 2006). More recently, the first evidence of decreased osteoclast number and activity in human bone of imatinib-treated patients was shown (Vandyke et al. 2012). Despite secondary hyperparathyroidism, biochemical markers of osteoclast activity have, by and large, not been found to be elevated (O'Sullivan et al. 2009). Long-term data will need to be accrued to show whether these cellular effects translate into altered clinical outcomes. Outcomes of interest include fracture risk and long-term side effects associated with potent antiresorptives such as atypical subtrochanteric femur fractures.

Osteoblasts Osteoblasts derive from human mesenchymal stem cells (MSCs) in the bone marrow and are responsible for de novo calcium phosphate deposition into the bone matrix while also regulating osteoclast differentiation and proliferation as shown in Fig. 1. Imatinib has been associated with mixed effects on osteoblast activity in vitro and in vivo. In vitro mineralization assays in osteoblast cell lines as well as human stromal cells show bone-forming activity with imatinib treatment (O'Sullivan et al. 2007, Fitter et al. 2008).
There is controversy whether patients undergoing imatinib treatment show altered levels of biochemical markers of osteoblast activity. A prospective study of imatinib treatment in CML patients showed transiently increased levels of P1NP and osteocalcin at 3 months, followed by a return to baseline levels, and finally a nonsignificant reduction in bone formation markers at 18 months (O'Sullivan et al. 2009, Vandyke et al. 2012). This study contrasts with the findings of the original crosssectional study and the subsequent prospective study, both by Berman et al. (2006), which showed chronic imatinib treatment was associated with decreased levels of osteocalcin without significant change in bone alkaline phosphatase (BAP). However, a key difference in these studies is the duration of treatment with imatinib - in the Berman study most patients were chronically treated. Rodent data have not supported a bone anabolic effect of imatinib (O'Sullivan et al. 2011). Most recently, bone histomorphometric prospective data in imatinib-treated patients showed, via serial iliac crest biopsy samples obtained at baseline and after 6,12 , and 24 months of treatment, i) no change in osteoblast numbers and ii) an increase in trabecular bone volume and trabecular thickness at 12 months but not at 24 months (the authors attributed the 24-month data to a lack of statistical power given that only eight patients had matched baseline and 24-month samples for analysis). The levels of BAP and P1NP were not significantly altered during imatinib treatment. At the 24 month mark, there was a trend toward reduction in both of these markers (Vandyke et al. 2012). Osteocalcin levels were not measured, because at baseline most patients had undetectably low levels. In vitro, imatinib appears to inhibit proliferation of human MSCs, promote early but not late osteoblast differentiation, and inhibit the development of fully mature osteoblasts (Zhang et al. 1998, Jönsson et al. 2012). BAP is synthesized in early osteoblast progenitors, and osteocalcin is synthesized by more mature osteoblasts; the findings reported with chronic imatinib treatment (normal BAP and low osteocalcin) are consistent with in vitro data. In this report, the effect of imatinib on osteoblast differentiation was shown to depend on the drug concentration and also on osteoblast maturity (Jönsson et al. 2012).

\section{Dasatinib}

Dasatinib is a TKI targeted at BCR-Abl kinase, but which possesses higher potency than imatinib. Like its predecessor, dasatinib has off-target kinase effects (most significantly SRC kinase blockade) that impact bone metabolism.

Published by Bioscientifica Ltd. 


\section{Effect on bone cells: osteoclasts and osteoblasts}

Dasatinib has been reported to inhibit osteoclastogenesis by inhibiting c-Fms osteoclast lineage progenitors (Fig. 1) and, indirectly, by inhibiting osteoblast-derived RANKL. In a rat model of physiologic bone turnover, it has been shown to increase trabecular bone volume likely via inhibition of osteoclast activity, while not having a significant effect on osteoblast parameters, suggesting that dasatinib may 'dysregulate' bone remodeling (Vandyke et al. 2010b). Another study has demonstrated direct MSC adipocyte differentiation at the expense of the osteoblast lineage (Borriello et al. 2011). This finding raises the potential for dasatinib to contribute to skeletal fragility as has been demonstrated with another class of drug, thiazolidinediones, that preferentially direct osteoblast differentiation toward adipocytes and increase fracture risk (Bilik et al. 2010). In contrast, in vivo data have shown increased osteoblastic differentiation from MSCs and increased osteoblastic activity, while also a downregulation of RANKL synthesis (Id Boufker et al. 2010). A final study showed differential effects on osteoblasts and osteoclasts leading to potential overlap of anabolism and resorption depending on drug concentration (Garcia-Gomez et al. 2012). Intriguingly, in this study, at low concentrations in mice, dasatinib was shown to favorably uncouple bone turnover and had an osteogenic effect as well as an inhibitory effect on osteoclasts.

According to the manufacturer's single-dose toxicity evaluation in monkeys, bone resorption was inhibited and a decrease in serum calcium and phosphorus were observed. These findings were reversible or partially reversible within the 14-day recovery period. In vitro, dasatanib potently inhibited PTH-stimulated osteoclastic bone resorption in fetal rat long bones. Also, dasatinib blocked the normalization of plasma calcium in a thyroparathyroidectomized rat model after PTH infusion (European Medicines Agency scientific discussion on dasatinib p46, available at: http://www.ema.europa.eu/ ema/index.jsp?curl=pages/medicines/human/medicines/ 000709/human_med_001062.jsp).

In chemotherapy-naive men with metastatic castrateresistant prostate cancer (with bone metastases) and increasing prostate-specific antigen levels, dasatinib monotherapy was shown to significantly reduce urinary NTX (a marker of osteoclast activity) and BAP (a marker of osteoblast activity) in 51 and 59\% of patients respectively (Yu et al. 2011). Concomitant bisphosphonate therapy did not appear to affect these results. The bone 'phenotype' of prostate cancer patients with bone metastases generally involves markedly increased bone turnover with primarily osteoblastic metastases. In this setting, downregulation of unchecked bone turnover (and osteoblastic activity) at the site of the metastases is favorable; dasatinib may be useful. In theory, dasatinib might be expected to exhibit a temporal increase in bone formation markers followed by a return to baseline, as does imatinib. However, in the setting of metastatic prostate cancer, this does not appear to be the case given the aforementioned bone turnover marker data was obtained at 12 weeks, the point at which imatinib appears to increase bone formation markers.

\section{Altered calcium and phosphorus metabolism}

Hypocalcemia and hypophosphatemia during dasatinib were found in patients with CML resistant or intolerant to imatinib, with highest incidence in advanced phase (12 and 18\% respectively; Sprycelw (dasatanib) package insert p10, available at http://packageinserts.bms.com/pi/ pi_sprycel.pdf). Of patients with chronic phase CML, the incidence of hypocalcemia and hypophosphatemia was $<1$ and 10\% respectively. In vitro, dasatinib dose dependently inhibited PTH-stimulated release of ${ }^{45} \mathrm{Ca}$ into the medium of fetal rat long bones with an apparent $\mathrm{IC}_{50}$ of $2 \mathrm{nM}$. At $5 \mathrm{nM}$, dasatinib completely blocked PTHstimulated bone resorption in vitro. The drug also blocked the normalization of plasma calcium in the thyroparathyroidectomized rat model after infusion of $\mathrm{PTH}$, evidence of anti-resorptive activity.

With respect to prostate cancer, secondary hyperparathyroidism is common among men with advanced disease (Murray et al. 2001), presumably for the most part due to increased calcium deposition into osteoblastic metastases. Future studies evaluating PTH levels in patients on dasatinib should take this physiology into account. Prostate cancer patients may thus be especially prone to hypocalcemia in the setting of potent antiresorptive drugs (intravenous bisphosphonates (IVBPs) and denosumab). Given that dasatinib also appears to affect PTH action and have antiresorptive activity, the risk of hypocalcemia could be theoretically magnified. Currently, in Phase II trials as treatment for a variety of cancers, subsequent data will help to clarify direct (SRC-mediated) and potential indirect (i.e. phosphate metabolism mediated) effects of dasatinib in comparison to imatinib on bone and mineral metabolism.

\section{SRC inhibitor: saracatanib}

Saracatanib is a newer ATP-competitive inhibitor of SRC kinase developed as targeted therapy for osteoporosis. In a Phase 1 trial carried out in healthy men, saracatinib reliably decreased biochemical markers of bone resorption by up to $88 \%$ from baseline after $24 \mathrm{~h}$ with no effect on bone

Published by Bioscientifica Ltd. 
formation markers and without serious adverse events (Hannon et al. 2010). The reported decrease in biochemical markers of bone resorption (CTX and UNTx) is comparable with that observed in studies of bisphosphonates in postmenopausal women. A study in patients with advanced solid malignancies metastatic to bone and resistant to standard treatment demonstrated significant dose-related reductions in bone resorption markers; there was a decrease in the bone formation marker, P1NP, but not in BAP (Hannon et al. 2012). Current Phase 1 trials in solid-tumor cancer patients with bone metastases will provide insights as to whether this drug will be useful in the setting of metastatic bone disease via its apparent anti-resorptive effects.

\section{VEGF inhibitors}

Following the success of imatinib in delivering effective oral treatment for CML, multiple ATP mimetic smallmolecule TKIs were developed for targets associated with multiple malignancies. VEGF has a key role in the osteoanabolic response (Towler 2003). The following description will focus on the primary targets of the described TKIs; nonetheless, therapeutic and off-target effects of multi-tyrosine kinase inhibition impart added potency and potential side effects to these agents. Within the VEGF inhibitor family, we shall focus on sunitinib and sorafenib as targets with reported bone metabolism endpoints.

Sunitinb Sunitinib is a TKI, targeting among other kinases VEGFR1-3, PDGFRa and b, FLT3, CSR, and RET. This agent is currently approved for GIST after disease progression on or after intolerance to imatinib mesylate; for advanced renal cell carcinoma; and for progressive, well-differentiated pancreatic neuroendocrine tumors (pNET) in patients with unresectable locally advanced or metastatic disease (Demetri et al. 2006, Motzer et al. 2012). Its mechanism of action is felt to be blockade of angiogenesis in the aforementioned tumors through VEGF-signaling disruption. Within the renal cell carcinoma population, there is probably a higher incidence of osteonecrosis of the jaw when IVBP therapy for bone metastases is combined with sunitinib (or sorafenib) than when IVBP is used alone; the mechanism for the increased risk remains unclear (Beuselinck et al. 2012). Possible etiologies for osteonecrosis of the jaw as a side effect in this population relate to the anti-angiogenic effects of sunitinib or antiresorptive activity, the latter which has been demonstrated by Sahi et al. in a small number of patients with RCC metastatic to bone and in a study of prostate cancer patients metastatic to bone (Dror Michaelson et al. 2009, Sahi et al. 2009, Agrillo et al. 2012, Baldazzi et al. 2012, Beuselinck et al. 2012).

Altered calcium and phosphorus metabolism In a prospective evaluation of renal cell carcinoma in patients beginning treatment with sunitinib, new onset hyperparathyroidism with stable calcium and phosphorus levels has recently been demonstrated (Baldazzi et al. 2012). Serum creatinine levels were reported as normal, although GFR was not calculated. Eighteen of 26 patients (69.2\%) developed hyperparathyroidism with normal serum calcium levels. The increase in PTH developed after a mean of 2.2 cycles of sunitinib (range, one to six cycles); after the drug was discontinued, in over 2-4 months, PTH levels returned to normal in all hyperparathyroid patients. As expected in the setting of hyperparathyroidism, there was a trend for increased 1,25dihydroxyvitamin $\mathrm{D}_{3}$ levels. This study also found that patients with hyperparathyroidism had a marked reduction in 24-h urinary calcium values: mean urinary calcium, $20.3 \mathrm{mg} / 24 \mathrm{~h}$, with an average decrease from the baseline of $94.14 \%(P<0.001)$. The values for $24 \mathrm{~h}$ urinary phosphorus excretion were within normal limits and remain unchanged from baseline, although the fractional excretion of phosphorus was not calculated. Hypophosphatemia was observed in six patients, yet only three out of these six patients developed high PTH levels. The totality of this data again suggests inadequate vitamin $\mathrm{D}$ action because in theory, elevations in 1,25-dihydroxyvitamin $\mathrm{D}_{3}$ levels should augment gastrointestinal (GI) absorption of calcium and prevent the extremely low 24-h urine calcium values detected, even in the face of elevations in PTH levels. Either a direct TKI effect on vitamin D-mediated intestinal calcium absorption or a progressive diminution in oral calcium intake related to advanced cancer are the two explanations for the $24 \mathrm{~h}$ urine calcium findings. Alternatively, the authors hypothesized that sunitinib, similar to imatinib, might inhibit PDGFR on both osteoclasts and osteoblasts.

Finally, in a pNET Phase III study with sunitinib, there was a higher incidence of hypocalcemia and hypophosphatemia with sunitinib than with placebo (all grades hypocalcemia: $36 \%$ with sunitinib vs $22 \%$ with placebo; all grades hypophosphatemia $34 \%$ with sunitinib vs $19 \%$ with placebo). PTH levels and other metabolic bone parameters were not evaluated in this trial (Sutentw (sunitinib) package insert p29, available at: http://label ing.pfizer.com/ShowLabeling. aspx?id=607).

Sorafenib Sorafenib is a TKI, targeting multiple molecular targets; primarily VEGFR2 in addition to FLT3,

Published by Bioscientifica Ltd. 
PDGFR, and FGFR1. This agent is currently approved for treatment of metastatic renal cell carcinoma and advanced hepatocellular carcinoma, while also receiving attention as treatment of medullary and differentiated thyroid carcinoma (Cabanillas et al. 2010, Lam et al. 2010). Bone metastases from differentiated thyroid cancer appear to be less responsive to sorafenib than do other sites of metastases (Cabanillas et al. 2010). In the context of its primary indication for RCC, sorafenib is associated with improved outcomes in patients with unresectable disease that is metastatic to bone (Albiges et al. 2012).

Sorafenib has been shown to reduce the bone resorption marker, urinary NTX, in RCC metastatic to bone; although head-to-head comparison was not done, the magnitude of reduction was ostensibly less than that from IVBP and denosumab (Sahi et al. 2009). In addition, recent reports have suggested the utility of sorafenib for the treatment of unresectable high-grade osteosarcoma, a malignancy where excess osteoid is produced by the malignant cells (Grignani et al. 2012). Biochemical markers of bone turnover were not assessed in this study.

Sorafenib has been shown to induce muscle loss (sarcopenia) in patients with metastatic RCC (Antoun et al. 2010); this is of direct relevance to bone fragility given the emerging link between falls, fragility fractures, and sarcopenia (Lang et al. 2010).

\section{RET inhibitors}

Vandetanib Vandetanib is a member of a group of small-molecule TKIs with activity against the RET protooncogene and is approved by FDA for advanced MTC. In a double-blind trial of locally advanced or metastatic MTC with 2:1 randomization and a total of 330 patients enrolled, more patients had hypocalcemia in the drugtreated than placebo-treated groups (11 vs 3\%); hypophosphatemia was not reported. There was also a higher risk of QT prolongation (of uncertain etiology) in the vandetanib-treated patients vs placebo ( 8 vs $1 \%$; Wells et al. 2012). QT prolongation, torsades de pointes, and sudden death are included in a boxed warning on the drug-prescribing information; electrolytes are to be periodically monitored. In theory, patients with $M E N$ related hyperparathyroidism should be less likely to become hypocalcemic and more likely to become hypophosphatemic; this sub-analysis was not reported. Wells et al. (2012) included eight of 19 patients with locally advanced metastatic cancer with bone metastases, with notable effects on tumor regression and no observed effects on renal or calcium metabolism. However, in patients with locally advanced metastatic-differentiated thyroid cancer, vandetanib was found to increase vitamin D and calcium replacement requirements, cause no change in calcium levels, yet still did significantly increase PTH and 1,25(OH)(2) vitamin D levels. The authors noted that this suggested a decreased intestinal absorption of vitamin D or lack of sun exposure as a result of photosensitization (Brassard et al. 2011). Ten of 39 patients in this study (all MTC patients) had postoperative hypoparathyroidism. The authors carried out a subanalysis on the hypoparathyroid patients and did not find significant changes in metabolic bone assays. It is probable that these patients would not be able to increase their PTH levels and thus be more vulnerable to hypocalcemia after treatment with vandetanib. In addition, clinically important hypocalcemia was possibly avoided via an increase in calcium and vitamin D supplementation. The values for $24 \mathrm{~h}$ urinary calcium levels were assessed only after patients were on drug and in general were unremarkable. Calcium absorption studies before and after drug treatment might help clarify whether vandetanib directly decreased intestinal calcium and vitamin $\mathrm{D}$ absorption and hence increased PTH levels. Decreased gut calcium absorption could also contribute to the observed drop in 25-OH D levels. Alternatively, the authors postulate that the findings could be due to a decrease in vitamin $\mathrm{D}$ action and note that, in their experience, severe hypercalcemia may occur if the 'very high' vitamin D doses required for sufficiency are not decreased when the TKI is stopped. Osteocalcin levels decreased after vandetanib treatment; resorption markers were not reported. Despite little or no cross-talk of the targeted tyrosine kinases by vandetanib, sunitinib, and imatinib inhibition, Brassard et al. observe increased levels of PTH, lower 25-OH D, and increased 1,25-OH D, suggesting a class effect. Long-term studies will be needed to assess metabolic bone effects of vandetanib given an otherwise tolerable side-effect profile.

Cabozantinib Cabozantinib (XL-184) is a nonselective RET inhibitor additionally targeting VEGFR2 and MET and currently approved by FDA for metastatic MTC (Kurzrock et al. 2011). Thyroid cancer may metastasize to bone in aggressive differentiated histologies or MTC, with preferential sites including vertebrae, pelvis, ribs, and femur, while portending poor prognosis in terms of survival and conveying a high risk of skeletal-related events (Kebebew et al. 2000, Pittas et al. 2000). Cabozantinib has additionally been studied in the context of castrate-resistant prostate cancer with metastatic bone involvement as part of a trial including advanced cancers (NCT00940225;

Published by Bioscientifica Ltd 
Dayyani et al. 2011). In this context, 55\% of patients with metastatic castrate-resistant prostate cancer experienced $>50 \%$ reduction in UNTx and $56 \%$ of patients experienced $>50 \%$ reduction in alkaline phosphatase. Recently, the results of a randomized Phase II study revealed that $72 \%$ or patients had regression of soft tissue lesions and $68 \%$ of evaluable patients had improvement on 99Tc-methylene diphosphonate bone scan. Random assignment was halted early based on the activity of cabozantinib/improvement in progression-free survival. Bone turnover markers (total alkaline phosphatase and C-terminal telopeptide) decreased $\geq 50$ in $57 \%$ of evaluable patients. Subjective improvement in bone pain was seen in $67 \%$ of subjects (79 of 116) with bone metastasis and complete resolution in 14 patients and $56 \%$ decreased or stopped narcotic usage (Smith et al. 2013). The bone scan represents the osteoblastic response to the tumor, and thus it is possible that cabozantinib has effects on both tumor and the bone microenvironment. Preclinical studies lend plausibility to theoretical effects of cabozantinib on the bone microenvironment; the hepatocyte growth factor (HGF)/MET pathway, which is targeted by cabozantinib, has been shown to affect expression in osteoblasts (Chen et al. 2012). Two Phase III trials have been initiated in men with progressive disease as a treatment for bone metastases from castrate-resistant prostate cancer. The dual activity of cabozantinib for RET and VEGFR2 makes it an attractive agent for multiple cancer types and it is undergoing early trials in a wide variety of cancers including pancreatic, sarcoma, non-small-cell lung cancer, and breast cancer. On the basis of the above limited data, cabozantinib does appear to affect bone mineral metabolism and needs further exploration.

\section{Summary}

The literature reviewed shows the promise of TKIs as a targeted therapy for multiple malignancies, while highlighting the importance of understanding the respective signaling pathways to anticipate potential side effects as summarized in Table 1. Even TKIs that do not have target receptors in common appear to have similar effects on bone metabolism, suggesting to an extent a class effect. In the case of imatinib, off-target kinase effects account for cellular effects leading to decreased osteoclastogenesis, possibly decreased overall bone turnover, as well as alterations in calcium and phosphate metabolism. It is difficult to discern the effect of chronic imatinib (or other TKI) therapy on risk for fragility fractures; no clear safety signal has emerged. Patients who are concomitantly receiving potent antiresorptive medications (bisphoshphonates and denosumab) and TKIs that have potent inhibitory effects on osteoclast activity should be monitored for potential complications known to occur with the latter: acutely, hypocalcemia, and over the long term, osteonecrosis of the jaw and atypical subtrochanteric fractures. Most newer TKIs are being studied in the setting of advanced cancers, many of which can metastasize to bone. Thus, bone issues pertinent to a long-term therapy with imatinib for chronic-phase CML, such as long-term osteoporotic fracture risk, are ostensibly less relevant to such patients with a limited life expectancy bone morbidity due to skeletal-related events has greater importance. We make note that the common TKI side effect of secondary hyperparathyroidism, classically thought to be a metabolic issue related to osteoporotic fracture risk, has recently been shown to have possible

Table 1 Summary of tyrosine kinase inhibitor (TKIs) effects on bone metabolism at the cellular and macroscopic level

\begin{tabular}{|c|c|c|c|}
\hline Inhibitor & Osteoclast effects & Osteoblast effects & Resorption \\
\hline Imatinib & $\begin{array}{l}\downarrow \downarrow \text {, Berman et al. (2006) } \\
\quad \text { and Vandyke et al. (2012) }\end{array}$ & $\begin{array}{l}\text { - }^{a}, \text { O'Sullivan et al. (2011), } \\
\text { Vandyke et al. (2012) and } \\
\text { Berman et al. (2013) }\end{array}$ & $\downarrow$, Berman et al. (2013) \\
\hline Dasatinib & $\begin{array}{l}\downarrow, \text { Garcia-Gomez et al. } \\
(2012)\end{array}$ & $-^{a}$, Borriello et al. (2011) & $\downarrow^{b}$, Yu et al. (2011) \\
\hline Saracatanib & $\downarrow$, Hannon et al. (2010) & & $\downarrow$, Hannon et al. (2010) \\
\hline & & & $\downarrow^{b}$, Hannon et al. (2012) \\
\hline Sunitinib & $\downarrow^{b}$, Maita et al. (2012) & & $\begin{array}{l}\downarrow^{b}, \text { Sahi et al. (2009), Dror } \\
\text { Michaelson et al (2009), } \\
\text { Maita et al. (2012), }\end{array}$ \\
\hline $\begin{array}{l}\text { Sorafenib } \\
\text { Vandetanib }\end{array}$ & & & $\downarrow^{b}$, Sahi et al. (2009) \\
\hline Cabozantinib & $\downarrow$, Dai et al. (2013) & $\downarrow$, Dai et al. (2013) & $\downarrow$, Dayyani et al. (2011) \\
\hline
\end{tabular}

Formation

- ${ }^{a}$, Vandyke et al. (2012)

$\downarrow^{b}$, Yu et al. (2011)

No change, Hannon et al. (2010)

$\downarrow^{\text {b }, ~ H a n n o n ~ e t ~ a l . ~(2012) ~}$

No change ${ }^{b}$, Dror

Michaelson et al. (2009)

$\downarrow$, Brassard et al. (2011)

$\downarrow$, Dayyani et al. (2011)

${ }^{a}$ Conflicting data.

${ }^{\mathrm{b}}$ Reported in metastatic disease to bone.

http://erc.endocrinology-journals.org DOI: 10.1530/ERC-12-0400
(C) 2014 Society for Endocrinology Printed in Great Britain
Published by Bioscientifica Ltd 
importance in the setting of zoledronic acid therapy for metastatic prostate carcinoma to bone - higher baseline PTH level was associated with decreased overall survival (Berruti et al. 2012). Newer classes of TKIs with potent antitumor effects will be likely associated with similar off-target effects on bone metabolism. In the setting of metastatic cancer to bone, the TKI class effect of osteoclast inhibition, combined with anti-tumor effects, theoretically should create synergy to lessen skeletal-related morbidity and mortality.

\section{Declaration of interest}

Dr A Farooki has consulted for Bayer Pharmaceuticals. The other authors declare no competing interests.

\section{Funding}

This review did not receive any specific grant from any funding agency in the public, commercial or not-for-profit sector.

\section{References}

Agrillo A, Nastro Siniscalchi E, Facchini A, Filiaci F \& Ungari C 2012 Osteonecrosis of the jaws in patients assuming bisphosphonates and sunitinib: two case reports. European Review for Medical and Pharmacological Sciences 16 952-957.

Albiges L, Oudard S, Negrier S, Caty A, Gravis G, Joly F, Duclos B, Geoffrois L, Rolland F, Guillot A et al. 2012 Complete remission with tyrosine kinase inhibitors in renal cell carcinoma. Journal of Clinical Oncology 30 482-487. (doi:10.1200/JCO.2011.37.2516)

Ando W, Hashimoto J, Nampei A, Tsuboi H, Tateishi K, Ono T, Nakamura N, Ochi T \& Yoshikawa H 2006 Imatinib mesylate inhibits osteoclastogenesis and joint destruction in rats with collagen-induced arthritis (CIA). Journal of Bone and Mineral Metabolism 24 274-282. (doi:10.1007/ s00774-006-0684-1)

Antoun S, Birdsell L, Sawyer MB, Venner P, Escudier B \& Baracos VE 2010 Association of skeletal muscle wasting with treatment with sorafenib in patients with advanced renal cell carcinoma: results from a placebo-controlled study. Journal of Clinical Oncology 28 1054-1060. (doi:10.1200/JCO.2009.24.9730)

Baldazzi V, Tassi R, Lapini A, Lunghi A, Garofoli E, Caruso S, Carini M \& Mazzanti R 2012 Sunitinib-induced hyperparathyroidism: a possible mechanism to altered bone homeostasis. Cancer 118 3165-3172. (doi:10.1002/cncr.26435)

Berman E, Nicolaides M, Maki RG, Fleisher M, Chanel S, Scheu K, Wilson BA, Heller G \& Sauter NP 2006 Altered bone and mineral metabolism in patients receiving imatinib mesylate. New England Journal of Medicine 354 2006-2013. (doi:10.1056/NEJMoa051140)

Berman E, Girotra M, Cheng C, Chanel S, Maki R, Shelat M, Strauss HW, Fleisher M, Heller G \& Farooki A 2013 Effect of long term imatinib on bone in adults with chronic myelogenous leukemia and gastrointestinal stromal tumors. Leukemia Research 37 790-794. (doi:10.1016/ j.leukres.2013.02.005)

Berruti A, Cook R, Saad F, Buttigliero C, Lipton A, Tampellini M, Lee KA, Coleman RE \& Smith MR 2012 Prognostic role of serum parathyroid hormone levels in advanced prostate cancer patients undergoing zoledronic acid administration. Oncologist 17 645-652. (doi:10.1634/ theoncologist.2011-0448)
Beuselinck B, Wolter P, Karadimou A, Elaidi R, Dumez H, Rogiers A, Van Cann T, Willems L, Body JJ, Berkers J et al. 2012 Concomitant oral tyrosine kinase inhibitors and bisphosphonates in advanced renal cell carcinoma with bone metastases. British Journal of Cancer $\mathbf{1 0 7}$ 1665-1671. (doi:10.1038/bjc.2012.385)

Bilik D, McEwen LN, Brown MB, Pomeroy NE, Kim C, Asao K, Crosson JC, Duru OK, Ferrara A, Hsiao VC et al. 2010 Thiazolidinediones and fractures: evidence from translating research into action for diabetes. Journal of Clinical Endocrinology and Metabolism 95 4560-4565. (doi:10.1210/jc.2009-2638)

Borriello A, Caldarelli I, Basile MA, Bencivenga D, Tramontano A, Perrotta S, Della Ragione F \& Oliva A 2011 The tyrosine kinase inhibitor dasatinib induces a marked adipogenic differentiation of human multipotent mesenchymal stromal cells. PLoS ONE 6 e28555. (doi:10.1371/journal. pone.0028555)

Brassard M, Neraud B, Trabado S, Salenave S, Brailly-Tabard S, Borget I, Baudin E, Leboulleux S, Chanson P, Schlumberger M et al. 2011 Endocrine effects of the tyrosine kinase inhibitor vandetanib in patients treated for thyroid cancer. Journal of Clinical Endocrinology and Metabolism 96 2741-2749. (doi:10.1210/jc.2010-2771)

Buchdunger E, Zimmermann J, Mett H, Meyer T, Müller M, Druker BJ \& Lydon NB 1996 Inhibition of the Abl protein-tyrosine kinase in vitro and in vivo by a 2-phenylaminopyrimidine derivative. Cancer Research 56 100-104.

Cabanillas ME, Waguespack SG, Bronstein Y, Williams MD, Feng L, Hernandez M, Lopez A, Sherman SI \& Busaidy NL 2010 Treatment with tyrosine kinase inhibitors for patients with differentiated thyroid cancer: the M. D. Anderson experience. Journal of Clinical Endocrinology and Metabolism 95 2588-2595. (doi:10.1210/jc.2009-1923)

Chen HT, Tsou HK, Chang CH \& Tang CH 2012 Hepatocyte growth factor increases osteopontin expression in human osteoblasts through PI3K, Akt, c-Src, and AP-1 signaling pathway. PLoS ONE 7 e38378. (doi:10.1371/journal.pone.0038378)

Cher ML, Towler DA, Rafii S, Rowley D, Donahue HJ, Keller E, Herlyn M, Cho EA \& Chung LW 2006 Cancer interaction with the bone microenvironment: a workshop of the National Institutes of Health Tumor Microenvironment Study Section. American Journal of Pathology 168 1405-1412. (doi:10.2353/ajpath.2006.050874)

Dai J, Zhang H, Karatsinides A, Keller JM, Kozloff K, Aftab DT, Schimmoller F \& Keller ET 2013 Cabozantinib inhibits prostate cancer growth and prevents tumor-induced bone lesions. Clinical Cancer Research 20 617-630.

Dayyani F, Gallick GE, Logothetis CJ \& Corn PG 2011 Novel therapies for metastatic castrate-resistant prostate cancer. Journal of the National Cancer Institute 103 1665-1675. (doi:10.1093/jnci/djr362)

Demetri GD, van Oosterom AT, Garrett CR, Blackstein ME, Shah MH, Verweij J, McArthur G, Judson IR, Heinrich MC, Morgan JA et al. 2006 Efficacy and safety of sunitinib in patients with advanced gastrointestinal stromal tumour after failure of imatinib: a randomised controlled trial. Lancet 368 1329-1338. (doi:10.1016/S0140-6736(06)69446-4)

Dewar AL, Cambareri AC, Zannettino ACW, Miller BL, Doherty KV, Hughes TP \& Lyons AB 2005 Macrophage colony-stimulating factor receptor c-fms is a novel target of imatinib. Blood 105 3127-3132. (doi:10.1182/ blood-2004-10-3967)

Dror Michaelson M, Regan MM, Oh WK, Kaufman DS, Olivier K, Michaelson SZ, Spicer B, Gurski C, Kantoff PW \& Smith MR 2009 Phase II study of sunitinib in men with advanced prostate cancer. Annals of Oncology 20 913-920. (doi:10.1093/annonc/mdp111)

Druker BJ, Tamura S, Buchdunger E, Ohno S, Segal GM, Fanning S, Zimmermann J \& Lydon NB 1996 Effects of a selective inhibitor of the $\mathrm{Abl}$ tyrosine kinase on the growth of Bcr-Abl positive cells. Nature Medicine 2 561-566. (doi:10.1038/nm0596-561)

El Hajj Dib I, Gallet M, Mentaverri R, Sévenet N, Brazier M \& Kamel S 2006 Imatinib mesylate (Gleevec) enhances mature osteoclast apoptosis and suppresses osteoclast bone resorbing activity. European Journal of Pharmacology 551 27-33. (doi:10.1016/j.ejphar.2006.09.007) 
Fitter S, Dewar AL, Kostakis P, To LB, Hughes TP, Roberts MM, Lynch K, Vernon-Roberts B \& Zannettino ACW 2008 Long-term imatinib therapy promotes bone formation in CML patients. Blood $\mathbf{1 1 1}$ 2538-2547. (doi:10.1182/blood-2007-07-104281)

François H, Coppo P, Hayman JP, Fouqueray B, Mougenot B \& Ronco P 2008 Partial Fanconi syndrome induced by imatinib therapy: a novel cause of urinary phosphate loss. American Journal of Kidney Diseases 51 298-301. (doi:10.1053/j.ajkd.2007.10.039)

Garcia-Gomez A, Ocio EM, Crusoe E, Santamaria C, Hernández-Campo P, Blanco JF, Sanchez-Guijo FM, Hernández-Iglesias T, Briñón JG, FisacHerrero RM et al. 2012 Dasatinib as a bone-modifying agent: anabolic and anti-resorptive effects. PLOS ONE 7 e34914. (doi:10.1371/journal. pone.0034914)

Gattei V, Celetti A, Cerrato A, Degan M, De Iuliis A, Rossi FM, Chiappetta G, Consales C, Improta S, Zagonel V et al. 1997 Expression of the RET receptor tyrosine kinase and GDNFR- $\alpha$ in normal and leukemic human hematopoietic cells and stromal cells of the bone marrow microenvironment. Blood 89 2925-2937.

Goodkin DA \& Pierce GF 1993 Role of platelet-derived growth factor in osteoblast function and bone synthesis. Wound Repair and Regeneration 1 203-212. (doi:10.1046/j.1524-475X.1993.10405.x)

Grignani G, Palmerini E, Dileo P, Asaftei SD, D'Ambrosio L, Pignochino Y, Mercuri M, Picci P, Fagioli F, Casali PG et al. 2012 A phase II trial of sorafenib in relapsed and unresectable high-grade osteosarcoma after failure of standard multimodal therapy: an Italian Sarcoma Group study. Annals of Oncology 23 508-516. (doi:10.1093/annonc/mdr151)

Hannon RA, Clack G, Rimmer M, Swaisland A, Lockton JA, Finkelman RD \& Eastell R 2010 Effects of the Src kinase inhibitor saracatinib (AZD0530) on bone turnover in healthy men: a randomized, double-blind, placebocontrolled, multiple-ascending-dose phase I trial. Journal of Bone and Mineral Research 25 463-471. (doi:10.1359/jbmr.090830)

Hannon RA, Finkelman RD, Clack G, Iacona RB, Rimmer M, Gossiel F, Baselga J \& Eastell R 2012 Effects of Src kinase inhibition by saracatinib (AZD0530) on bone turnover in advanced malignancy in a phase I study. Bone 50 885-892. (doi:10.1016/j.bone.2011.12.017)

Hock JM 1994 Platelet-derived growth factor enhances bone cell replication, but not differentiated function of osteoblasts. Endocrinology 134 1423-1428.

Horowitz MC, Coleman DL, Flood PM, Kupper TS \& Jilka RL 1989 Parathyroid hormone and lipopolysaccharide induce murine osteoblast-like cells to secrete a cytokine indistinguishable from granulocytemacrophage colony-stimulating factor. Journal of Clinical Investigation 83 149-157. (doi:10.1172/JCI113852)

Huang EJ, Nocka KH, Buck J \& Besmer P 1992 Differential expression and processing of two cell associated forms of the kit-ligand: KL-1 and KL-2. Molecular Biology of the Cell 3 349-362. (doi:10.1091/mbc.3.3.349)

Ianotto JC, Tempescul A, Amet Y, Grall P, Dalbies F, Eveillard JR, Guillerm G \& Berthou C 2012 Imatinib mesylate induces massive and nonspecific aminoaciduria in CML patients. American Journal of Hematology 87 437-439. (doi:10.1002/ajh.23113)

Id Boufker H, Lagneaux L, Najar M, Piccart M, Ghanem G, Body JJ \& Journé F 2010 The Src inhibitor dasatinib accelerates the differentiation of human bone marrow-derived mesenchymal stromal cells into osteoblasts. BMC Cancer 10 298. (doi:10.1186/1471-2407-10-298)

IOM 2011 In Dietary Reference Intakes for Calcium and Vitamin D. Washington, DC, USA: National Academies Press.

Jönsson S, Hjorth-Hansen H, Olsson B, Wadenvik H, Sundan A \& Standal T 2012 Imatinib inhibits proliferation of human mesenchymal stem cells and promotes early but not late osteoblast differentiation in vitro. Journal of Bone and Mineral Metabolism 30 119-123. (doi:10.1007/ s00774-011-0323-3)

Kebebew E, Ituarte PH, Siperstein AE, Duh QY \& Clark OH 2000 Medullary thyroid carcinoma: clinical characteristics, treatment, prognostic factors, and a comparison of staging systems. Cancer 88 1139-1148. (doi:10.1002/(SICI)1097-0142(20000301)88:5 < 1139::AID-CNCR26> 3.0.CO;2-Z)
Kurzrock R, Sherman SI, Ball DW, Forastiere AA, Cohen RB, Mehra R, Pfister DG, Cohen EE, Janisch L, Nauling F et al. 2011 Activity of XL184 (Cabozantinib), an oral tyrosine kinase inhibitor, in patients with medullary thyroid cancer. Journal of Clinical Oncology $\mathbf{2 9}$ 2660-2666. (doi:10.1200/JCO.2010.32.4145)

Lam ET, Ringel MD, Kloos RT, Prior TW, Knopp MV, Liang J, Sammet S, Hall NC, Wakely PE, Vasko VV et al. 2010 Phase II clinical trial of sorafenib in metastatic medullary thyroid cancer. Journal of Clinical Oncology 28 2323-2330. (doi:10.1200/JCO.2009.25.0068)

Lang T, Streeper T, Cawthon P, Baldwin K, Taaffe DR \& Harris TB 2010 Sarcopenia: etiology, clinical consequences, intervention, and assessment. Osteoporosis International 21 543-559. (doi:10.1007/s00198-0091059-y)

Maes C, Carmeliet G \& Schipani E 2012 Hypoxia-driven pathways in bone development, regeneration and disease. Nature Reviews. Rheumatology 8 358-366. (doi:10.1038/nrrheum.2012.36)

Maita S, Yuasa T, Tsuchiya N, Mitobe Y, Narita S, Horikawa Y, Hatake K, Fukui I, Kimura S, Maekawa T \& Habuchi T 2012 Antitumor effect of sunitinib against skeletal metastatic renal cell carcinoma through inhibition of osteoclast function. International Journal of Cancer 130 677-684. (doi:10.1002/ijc.26034)

Motzer RJ, Hutson TE, Olsen MR, Hudes GR, Burke JM, Edenfield WJ, Wilding G, Agarwal N, Thompson JA, Cella D et al. 2012 Randomized phase II trial of sunitinib on an intermittent versus continuous dosing schedule as first-line therapy for advanced renal cell carcinoma. Journal of Clinical Oncology 30 1371-1377. (doi:10.1200/JCO.2011. 36.4133)

Murray RM, Grill V, Crinis N, Ho PW, Davison J \& Pitt P 2001 Hypocalcemic and normocalcemic hyperparathyroidism in patients with advanced prostatic cancer. Journal of Clinical Endocrinology and Metabolism 86 4133-4138. (doi:10.1210/jcem.86.9.7864)

O'Sullivan S, Naot D, Callon K, Porteous F, Horne A, Wattie D, Watson M, Cornish J, Browett P \& Grey A 2007 Imatinib promotes osteoblast differentiation by inhibiting PDGFR signaling and inhibits osteoclastogenesis by both direct and stromal cell-dependent mechanisms. Journal of Bone and Mineral Research 22 1679-1689. (doi:10.1359/jbmr. 070719)

O'Sullivan S, Horne A, Wattie D, Porteous F, Callon K, Gamble G, Ebeling P, Browett P \& Grey A 2009 Decreased bone turnover despite persistent secondary hyperparathyroidism during prolonged treatment with imatinib. Journal of Clinical Endocrinology and Metabolism 94 1131-1136. (doi:10.1210/jc.2008-2324)

O'Sullivan S, Naot D, Callon KE, Watson M, Gamble GD, Ladefoged M, Karsdal MA, Browett P, Cornish J \& Grey A 2011 Imatinib mesylate does not increase bone volume in vivo. Calcified Tissue International 88 16-22. (doi:10.1007/s00223-010-9429-1)

Pausova Z, Soliman E, Amizuka N, Janicic N, Konrad EM, Arnold A, Goltzman D \& Hendy GN 1996 Role of the RET proto-oncogene in sporadic hyperparathyroidism and in hyperparathyroidism of multiple endocrine neoplasia type 2. Journal of Clinical Endocrinology and Metabolism 81 2711-2718.

Pittas AG, Adler M, Fazzari M, Tickoo S, Rosai J, Larson SM \& Robbins RJ 2000 Bone metastases from thyroid carcinoma: clinical characteristics and prognostic variables in one hundred forty-six patients. Thyroid $\mathbf{1 0}$ 261-268. (doi:10.1089/thy.2000.10.261)

Sahi C, Knox JJ, Hinder V, Deva S, Cole D \& Clemons MBR 2009 The effects of sorafenib and sunitinib on bone turnover markers in patients with bone metastases from renal cell carcinoma. American Society of Clinical Oncology 27 (supplement) e16145.

Schabel SI, Tyminski L, Holland RD \& Rittenberg GM 1980 The skeletal manifestations of chronic myelogenous leukemia. Skeletal Radiology 5 145-149. (doi:10.1007/BF00347255)

Schipani E, Maes C, Carmeliet G \& Semenza GL 2009 Regulation of osteogenesis-angiogenesis coupling by HIFs and VEGF. Journal of Bone and Mineral Research 24 1347-1353. (doi:10.1359/jbmr.090602) 
Sherman SI 2009 Advances in chemotherapy of differentiated epithelial and medullary thyroid cancers. Journal of Clinical Endocrinology and Metabolism 94 1493-1499. (doi:10.1210/jc.2008-0923)

Smith DC, Smith MR, Sweeney C, Elfiky AA, Logothetis C, Corn PG, Vogelzang NJ, Small EJ, Harzstark AL, Gordon MS et al. 2013 Cabozantinib in patients with advanced prostate cancer: results of a phase II randomized discontinuation trial. Journal of Clinical Oncology 31 412-419. (doi:10.1200/JCO.2012.45.0494)

Soriano P, Montgomery C, Geske R \& Bradley A 1991 Targeted disruption of the c-src proto-oncogene leads to osteopetrosis in mice. Cell $\mathbf{6 4}$ 693-702. (doi:10.1016/0092-8674(91)90499-O)

Steingrímsson E, Moore KJ, Lamoreux ML, Ferré-D’Amaré AR, Burley SK, Zimring DC, Skow LC, Hodgkinson CA, Arnheiter H \& Copeland NG 1994 Molecular basis of mouse microphthalmia (mi) mutations helps explain their developmental and phenotypic consequences. Nature Genetics 8 256-263. (doi:10.1038/ng1194-256)

Towler DA 2003 Angiogenesis and marrow stromal cell fates: roles in bone strength. Osteoporosis International 14 (Suppl 5) S46-S50 (discussion S50-S53). (doi:10.1007/s00198-003-1473-5)

Vandyke K, Fitter S, Dewar AL, Hughes TP \& Zannettino ACW 2010a Dysregulation of bone remodeling by imatinib mesylate. Blood $\mathbf{1 1 5}$ 766-774. (doi:10.1182/blood-2009-08-237404)

Vandyke K, Dewar AL, Diamond P, Fitter S, Schultz CG, Sims NA \& Zannettino ACW $2010 b$ The tyrosine kinase inhibitor dasatinib dysregulates bone remodeling through inhibition of osteoclasts in vivo. Journal of Bone and Mineral Research 25 1759-1770. (doi:10.1002/ jbmr.85)
Vandyke K, Fitter S, Drew J, Fukumoto S, Schultz CG, Sims NA, Yeung DT, Hughes TP \& Zannettino ACW 2012 Prospective histomorphometric and DXA evaluation of bone remodeling in imatinib-treated CML patients: evidence for site-specific skeletal effects. Journal of Clinical Endocrinology and Metabolism 98 67-76. (doi:10.1210/jc.2012-2426)

Wells SA, Robinson BG, Gagel RF, Dralle H, Fagin JA, Santoro M, Baudin E, Elisei R, Jarzab B, Vasselli JR et al. 2012 Vandetanib in patients with locally advanced or metastatic medullary thyroid cancer: a randomized, double-blind phase III trial. Journal of Clinical Oncology $\mathbf{3 0}$ 134-141. (doi:10.1200/JCO.2011.35.5040)

Yang K-H, Park S-Y, Park S-W, Lee S-H, Han S-B, Jung W-K \& Kim S-J 2010 Insufficient bilateral femoral subtrochanteric fractures in a patient receiving imatinib mesylate. Journal of Bone and Mineral Metabolism 28 713-718. (doi:10.1007/s00774-010-0203-2)

Yu EY, Massard C, Gross ME, Carducci MA, Culine S, Hudes G, Posadas EM, Sternberg CN, Wilding G, Trudel GC et al. 2011 Once-daily dasatinib: expansion of phase II study evaluating safety and efficacy of dasatinib in patients with metastatic castration-resistant prostate cancer. Urology 77 1166-1171. (doi:10.1016/j.urology.2011.01.006)

Zelzer E, McLean W, Ng Y-S, Fukai N, Reginato AM, Lovejoy S, D'Amore PA \& Olsen BR 2002 Skeletal defects in VEGF(120/120) mice reveal multiple roles for VEGF in skeletogenesis. Development 129 1893-1904.

Zhang Z, Chen J \& Jin D 1998 Platelet-derived growth factor (PDGF)-BB stimulates osteoclastic bone resorption directly: the role of receptor $\beta$. Biochemical and Biophysical Research Communications 251 190-194. (doi:10.1006/bbrc.1998.9412)

Received in final form 17 January 2014

Accepted 27 January 2014

Made available online as an Accepted Preprint

29 January 2014
(C) 2014 Society for Endocrinology Printed in Great Britain 